\title{
Augmentations of the Real A Critical Interrogation of the Relationship between the Actual, the Virtual and the Real
}

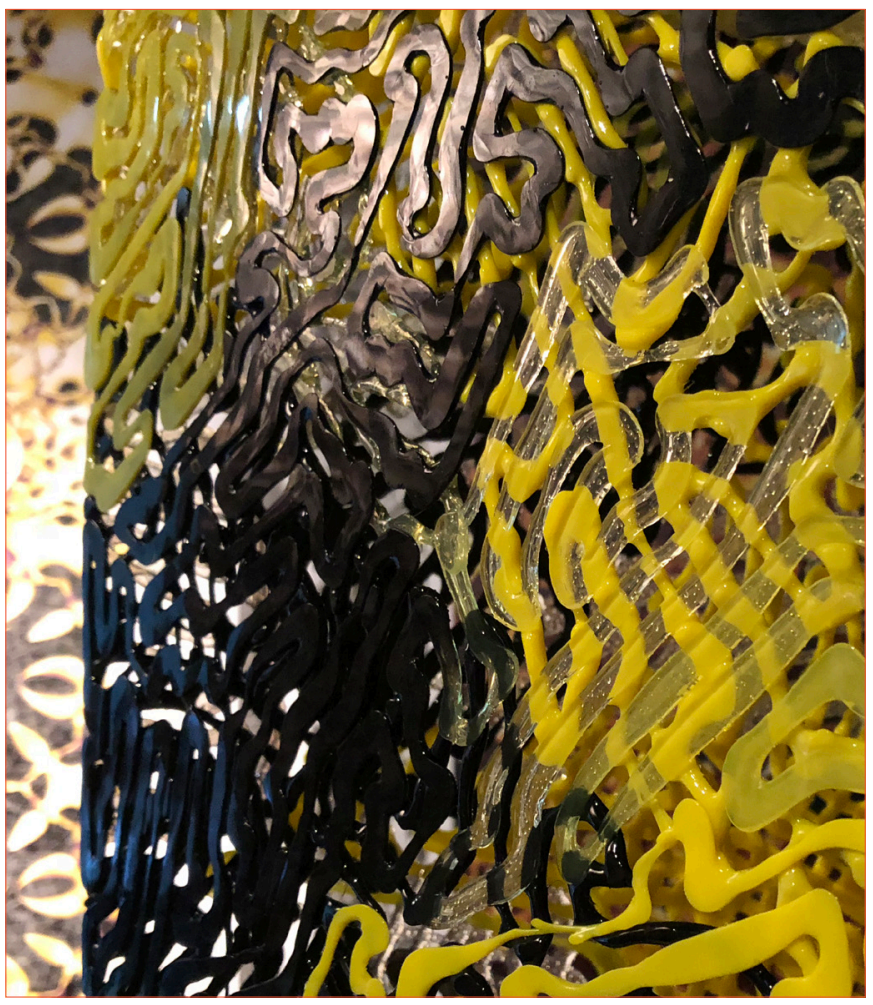

Figure 1. Closeup of the Digital Hypostyle as shown at the Digital Futures Exhibition Shanghai 2018

\section{COLUMNS, STYLITES, TOTEMS}

Augmentations of the Real presents itself as an occasion to critically interrogate the opportunities that Augmented Reality present for the discipline of architecture ${ }^{1}$. The problem was illuminated from different angles, reaching from aspects of the augmentation of spatial experiences through articulation and ornamentation, to aspects of $A R$ as an aid in advanced construction methodologies. Special attention was given to the fact that these techniques seamlessly fuse aspects of symbolic culture with considerations of materialism.

Augmentations of the Real is profoundly embedded in speculative territories. Moments of uncertainty collide with aspects of precision and control. The result is not an imitation of the former but rather a contemporary interpretation. The foundation can be discerned in the possibility to overlap various experience levels, which allows mining for potentialities in contemporary ornamentations. In this extent, Augmentations of the Real can be considered part of the discussion on PostDigital discourse in Architecture. An era in which computational tools are part of normal reality and other aspects of Digital Design are positioned center stage. Not the toolsets become the main actors, but the cultural agency produced by the toolsets.

I just mentioned other as being one of the goals of the examinaUniversity of Michigan tions of Augmentations of the Real. It is easy to propose other aspects as an option without going into detail, so instead of wading into vague hyperbole, I will explain it along the example used for the exhibition Digital Futures 2018. In this example the architectural icon of 
the column was un-shelved to serve as an object of contemplation. It is astounding that browsing through tomes of recent architecture theory, the column does not make an appearance even once ${ }^{2}$. Testament to the massive aversion towards the column demonstrated by modern, and post-modern movements. Two examples, under many, for this aversion come to mind: Coop Himmelblau's desire to erase the column, impressively demonstrated for example in their BMW-World ${ }^{3}$ building, and Peter Eisenman's use of the term Grid, which in fact can be read as intersecting colonnades - a series of columns penetrating through spatial volumes, as for example in the Wexner Center in Columbus Ohio ${ }^{4}$. Apart from the columns importance as a technical device in the architectural canon -negotiating the transport of loads from one floor to the next- it is loaded with symbolic value, without any technical efficacy. From the totemic poles of Native Americans to the Stylites ${ }^{5}$ of Christian mythology to the pillars of Hercules and the monumental Trajan Column in Rome - columns are deeply ingrained in the texture of architectures contribution to the ecology of symbolic culture ${ }^{6}$. As Manuel de Landa describes ${ }^{7}$ there can be a differentiation between material and symbolic culture, but they are both connected and influence each other like communicating vessels. In the same realm of thinking we can position Gottfried Semper, who's observations on the nature of columns and their origin can be considered inspirations for the work on the installation Augmentation of the Real. Semper's rigorous examination of the qualities of stereotomic constructions, as well as his well known feud over the fact that Trajan's column was originally colorfully paint$\mathrm{ed}^{8}$. Of course this presents an opportunity for an interesting provocation today. The Polychromic wars certainly inspired the colorful nature of the columns in the exhibition. As the subtitle of the installation already suggests the installation can be considered a critical interrogation of the relationship between the actual, the virtual and the real. Three distinct categories of thinking about the nature of our relationship to the rest of the universe and our possible, or impossible position therein. To explain all three categories in depth would fill an entire tome, but I will say this: As mentioned before, the project is aligned with considerations of a Postdigital age of production. In this universe of thinking, the discipline of architecture has surpassed the first cycle of obsession with digital toolsets and are searching for the cultural contribution to our contemporary age. In a world where digital tools are ubiquities (from autocorrect to Instagram filters to Al

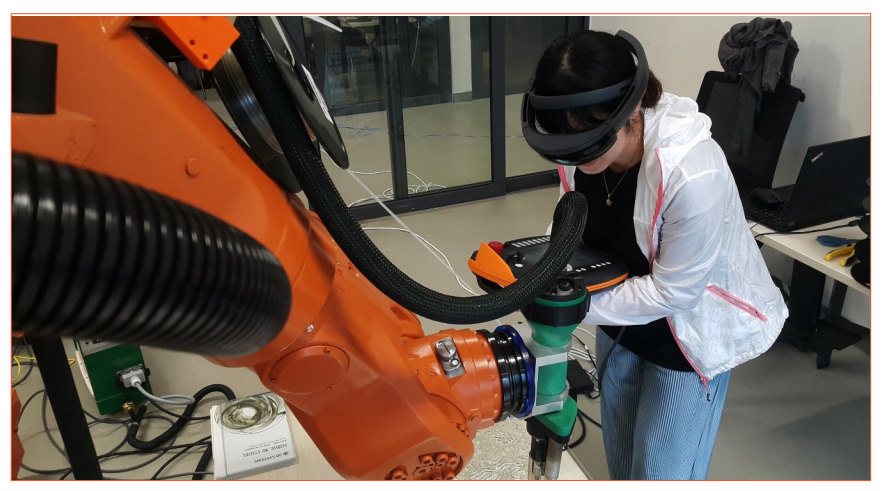

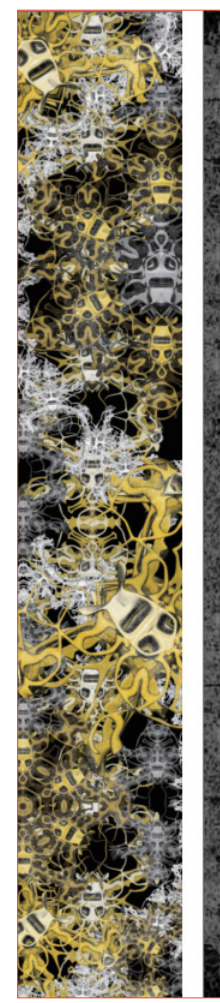
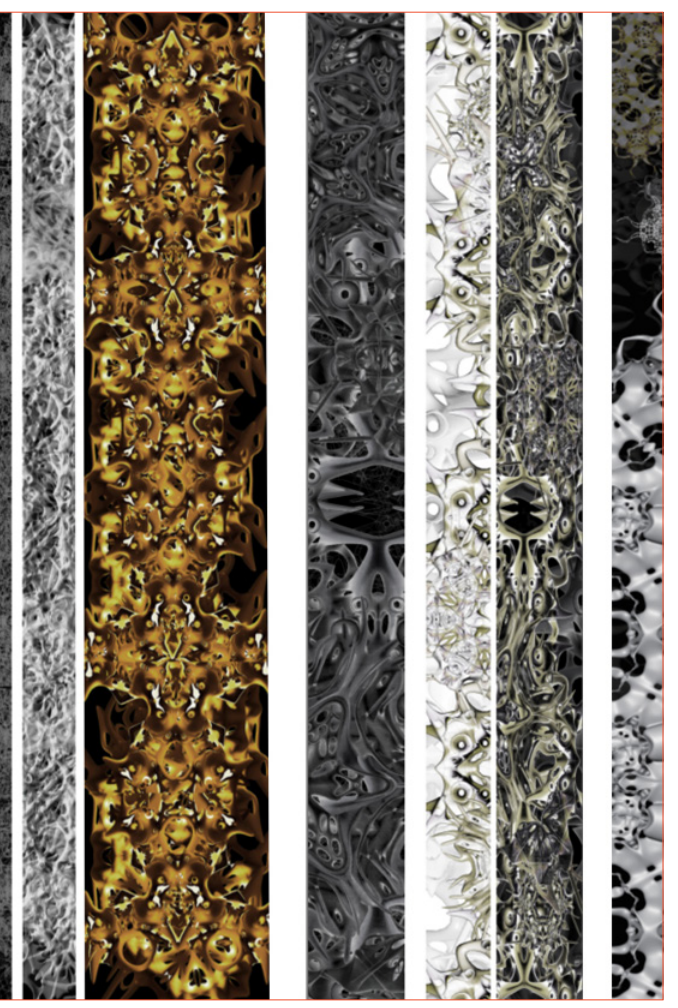

Figure 3. Series of patterns utilized as triggers for the AR application. The application could be used with conventional tablets and mobile phones.

robocalls that pass as humans) and cultural production is not a priori defined by the original piece of art -or originality for that matternovel tools of observation come into place. At the same time it allows to speculate about the value of the actual ${ }^{9}$, the virtual ${ }^{10}$ and the real ${ }^{11}$ Augmented Reality ${ }^{12}$ per se is defined by the application of symbolic gestures as interface between the material and the symbolic realm of computational environments. In a sense, Augmented Reality applications (Figure 2) propose a synthetic ecology that is primarily defined by their inherent properties, such as simulation, enhancement and intelligence gathering, overlapping two levels of information, which operate between physical environments, and computationally driven information.

The testbed for the examinations executed in Augmentations of the Real was found in the archetype of the column. Ornamented ${ }^{13} \mathrm{col}-$ umns have a long tradition as freestanding stela, specifically designed as memento, marker and memorial. The application of AR is able to extend the narrative qualities of the archetype of the column. The combination between one real concrete column, three ornamented columns and eighteen virtual columns produce a forest of columns, a weird hypostyle hall, oscillating between the actual, the real and the virtual.
Figure 2. Student comparing the toolpath printed by the robot with an overlaid virtual model of the toolpath 


\section{THE APPLICATION OF AR IN ROBOT-HUMAN FABRICATION}

In the previous section we described how Augmented Reality was utilized as a method of representation within the exhibition setting of the DigitalFutures exhibition at Tongji CAUP in July 2018. The application of AR with the use of conveniently available means, such as Tablets and Smartphones, opens up opportunities to create a spatial environment saturated by a multiplicious level of sensorial impulses or stimuli. This was only one part of the application of Augmented Reality (AR) in the context of the DigitalFutures exhibition. Augmented Reality describes a method in which the environment is still perceivable but is overlaid with 3D information, this of course opens up an entire array of possible applications, of which the use as representational tool is the most obvious, the most evident, and probably also the most boring one.

A far more interesting application can be found in the possibility to introduce AR applications to the construction site. The benefits of this move are quite evident. In the scenario where the architect has to convey complex information to a construction crew for example. By demonstrating the exact positioning of elements and components to the laymen the margin for error can be significantly lowered. This alone would justify the use of AR, however it goes beyond this, as applications such as Fologram do not only convey static information, but also allow to demonstrate processes. Meaning that the information seen through the Holographic device not only shows the final stage of a fabrication process, but also the way to get there. The workshop Augmentations of the Real, made use of the AR application Fologram in order to overlay virtual with real artifacts. Using a HoloLens Students were able to perceive the montage points for the panels (Figure 4).

\section{DIFFERENTIAL GROWTH ALGORITHM}

The panels were based on application of a space filling curve algorithm devised from Grasshopper. More specifically it was a differential growth algorithm that was applied on a simple rectangular plane in order to fill the space with a single line that never crossed itself. The main aim in avoiding self-inflections and a continuous line was to

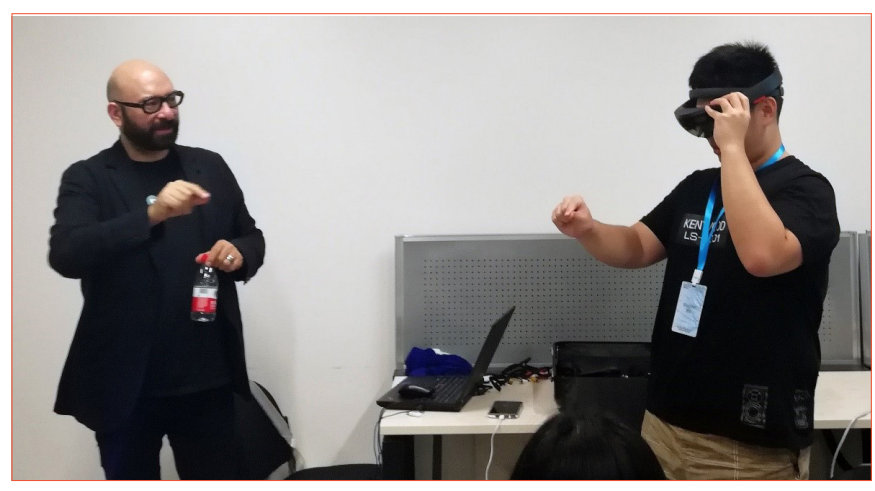

Figure 4. Teaching students the use of a HoloLens.

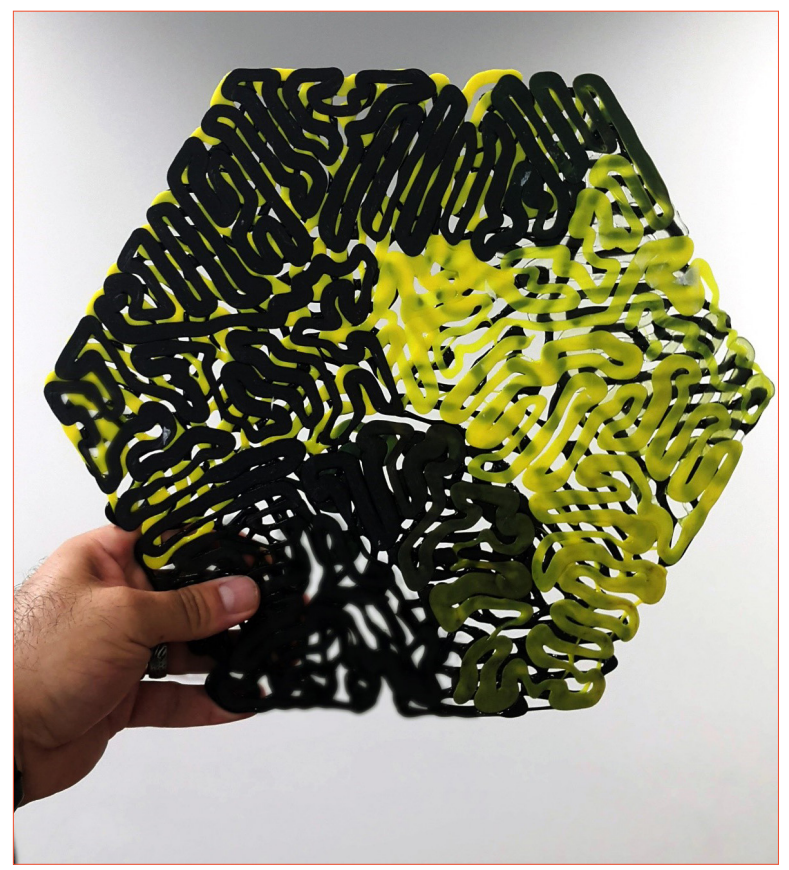

Figure 5. : test panel produced for the Salamander Column, DigitalFutures exhibition, Tongji CAUP 2018

develop a fabrication protocol that supports the use of fused deposition modeling without inflating areas of the deposition by overlapping the toolpath. Due to the fact that the path did not intersect, the integrity of the panel was not given. A single layer rather responded in a very elastic way. By applying two layers, in different directions, the stability increased profoundly (Figure 5). Providing a high integrity panel with a low material consumption. No specific structural analysis was done during the short workshop, but it certainly would be interesting to optimize the process by making the differen-tial growth algorithm response to specific pressures such as gravity, loads or wind pressures. This could be a result out of the workshop which would command further exploration in larger scale, for example for load bearing

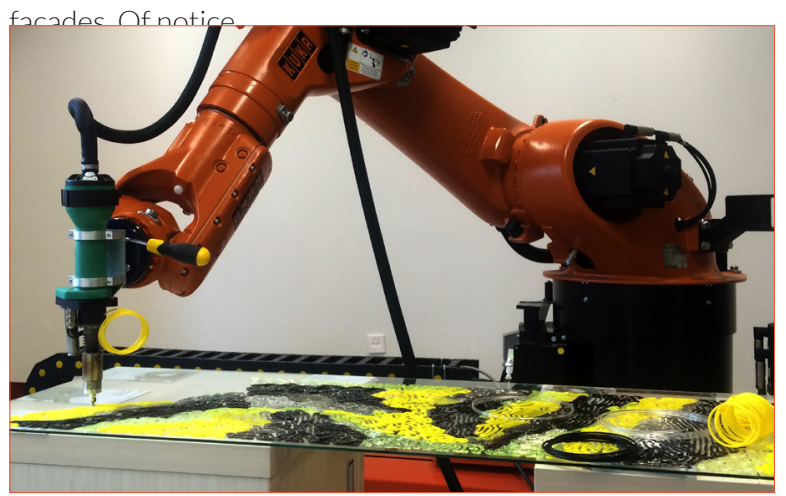

Figure 6. printing of the large shell panes of the Salamander Column. The glass pane ensured that the panels are straight and even 


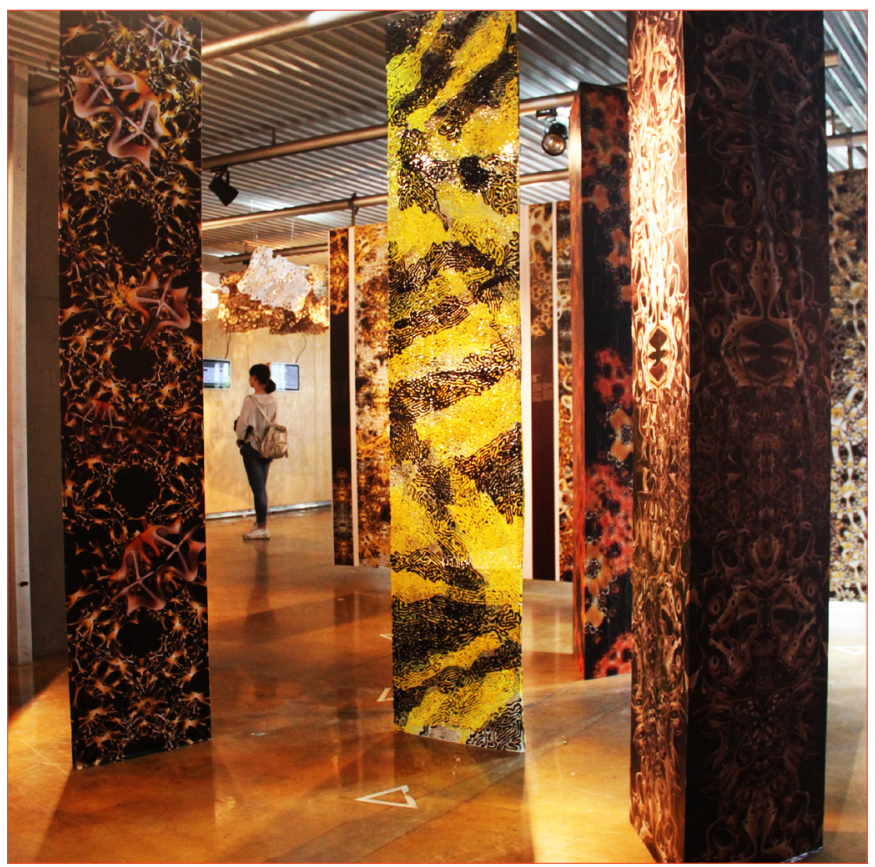

Figure 7. Final installation at Digital Futures Exhibition, Tongji, Shanghai, China, 2018

is also the use of coloration during the fabrication process. In recent years SPAN has been experimenting around with the use of continuously changing colorations in the fabrication process, as evidenced for example in Sandra Manninger's fabrication courses at Taubman College of Architecture and Urban Planning, or in Matias del Campo's studio at RMIT in Australia. In the case of the Augmented Realities workshop at Tongji a specific color palette was selected: Black, yellow and transparent. This combination allowed for multiplicious effects such as slow transitions between the colors, the gradient change from opaque to transparent etc. The color palette was also the inspiration for the name of the column, which we called the Salamander column. A Salamander is a small lizard like creature that populated the Austrian Alps and who's outstanding characteristic is the yellow and black spotted skin - a warning sign due to the toxicity of the skin covered in dangerous samandarin. To keep the production of the column under control considering the tight schedule the decision was made to reduce the column to a minimum of six components, consisting of three panels that constitute the shell of the column and three support fins in the inside. The consistent materiality and coloration ensured that all these components could be implemented in a seamless fashion.

The students produced the panels for the Salamander Column utilizing a Kuka KR 160 Robot outfitted with a Dohle Micro Extruder DX283 as end effector. The extruder acts akin to the extrusion head of a 3D printer, with the only main difference that diameter is much larger. The $3 \mathrm{~mm}$ extrusion nozzle is able to extrude around $600 \mathrm{~g}$ of Material per hour. In comparison to larger Dohle extruder heads it is not a large amount of material, but due to the morphology of the panels based on a continuous line defined by a differential growth algorithm, the material consumption was reduced to start with. So much so that it was possible to produce an entire panel for testing purposed without falling behind in the time schedule. One specific approach was to print the panels on a glass surface (Figure 6). The combination of thick glass pane as printing surface with a heated surface underneath reduced the risk of warping, so that the final result of the panels where straight and undistorted. Instead of utiliz-ing Augmented Reality as a mere mean of representation, this work-shop made use of AR as an aid in the construction of the column. This served as a proof of concept that to demonstrate the opportunities within AR as a methodology for the construction site. In the work-shop students were trained in the use of the Microsoft HoloLens for two main purposes.

1: To evaluate their designs of the virtual columns that were going up on display in the gallery

2: More importantly, to use the HoloLens as an aid in the construction of the 3D printed column.

The assembly of the column only took 3 hours as the Holograph helped in exactly positioning the supporting fins in 3-dimensional space. This ensured a precise assembly of the components in space.

\section{CONCLUSION}

In conclusion it can be stated that the workshop Augmentations of the Real served as a successful proof of concept for two specific criteria. On the one side the application of AR as a mode of exploration for the enhancement of spatial experiences, as exemplified in the virtual Hypostyle hall presented in the exhibition (Figure 7). The focus of this aspect of the application of $A R$ is on the potentialities as a mean of expression within three-dimensional space. The combina-tion between real, actual and virtual columns present themselves as a commentary on the lineage of the column as both technical mean of production as well as cultural signifier. The virtual column at the end of the day is most likely the epitome of a column as a pure cultural sig-nifier, rather than just a support structure. This approach allows for a critical interrogation of the column in our contemporary context, and more specifically within the realm of computational design,

The second criteria examine the use of AR within the construction site, by applying it in small scale in the fabrication and montage of a columns. Special attention is given in this case into the implementation of human ingenuity and pattern recognition talent within a robotic fabrication setup. The workshop participants used a HoloLens and Fologram setup to precisely position the components of the Salamander column. This ensured not only a precise setup of the components but also quick progress with a low error margin.

In a next step this approach will be applied to a more complex model, consisting of more components. The main aim however, is to apply this technique not only in the save environment of the fabrication lab-oratory, but also in the wild - the construction site. 


\section{Notes}

1. See also: The Materialism of ARCHITECTURAL AUTOMATIONS - A critical Interrogation of Automation, Accelerationism and Ornament. Matias del Campo \& Sandra Manninger 2018

2. Examples include: Nesbitt K. (ed.), Theorizing a New Agenda for Architecture, An Anthology of Architectural Theory 1965-1995, Sykes K.A. (ed.) Constructing a New Agenda, Architectural Theory 1993 -2009.

3. Coop Himmelb(I)au, BMW World, Munich, Germany 2007

4. Peter Eisenman, Wexner Center, Columbus, Ohio, 1989

5. Stylite, A stylite (from Greek, stylits, "pillar dweller", derived from, stylos, "pillar", Classical Syriac: as onáyé) or pillar-saint is a type of Christian ascetic who lives on pillars, preaching, fasting and praying. (retrieved from Wikipedia, https:// en.wikipedia.org/wiki/Stylite 11.06.2018)

6. Adolf Loos has made a contribution to this universe of thinking too: The columns in the entrance of the Haus am Michaelerplatz - a wonderful set of columns cut out of Euboean Cipollino marble- are only there to mark the entrance and have no structural function at all. The well trained architect's eye may notice right away that the striation of the marble is actually in the wrong direction, and that the columns would very likely crack vertically if stressed by loads.

7. Manuel de Landa in his Seminar ARCH Theory II: Philosophy of Materials and Structures, PennDesign, University of Pennsylvania.

8. Gottfried Semper, Der Stil in den technischen und tektonischen Künsten oder praktische Ästhetik: ein Handbuch für Techniker und Kunstfreunde (Band 2), Frankfurt a. M 1860, p. 399

9. Not surprisingly there are eight different terms when translating the term Actual into German: Eigentlich, Tatsächlich, Wirklich, Effektiv, Wahr, Real, Gegenwärtig, Derzeitig. All of which have a very distinct meaning attuned to specific situations, circumstances and lingual precision. In the frame of the considerations of this essay, I would argue that Eigentlich and Gegenwärtig fit the bill the best in that they describe a moment in the presence of our reality without penetrating the area of Realism, which has a distinct different meaning.

10. Virtual: In this realm of thinking the term Virtual references two worlds. On the one side the way the Henry Bergson and Gilles Deleuze considered the Virtual to be an approximation to an aspect of Reality that is Ideal, but remains just that: an aspect. Or in the words of Marcel Proust: Real but not Actual, Ideal but not Abstract. On the other it pretends to be as if it was real - but not in fact. A notion that fits well with the possibilities of contemporary computational design.

11. Real: As with the term Actual there are at least eight ways to translate the term Real into German. Echt, Real, Wahr,
Tatsächlich, Wirklich, Eigentlich, Richtiggehend, Effektiv. Echt and Wahr serve as auxiliary terms to explain the ambition of the usage of the term in the frame of this piece. Echt can be translated as real, authentic and embedded in the material experience of the world, versus Wahr denominates aspects of truthfulness, honesty and reliance. Somewhere between these two poles can be found a material truth to existence or Reality (Realität)

12. AR, Augmented Reality. An enhanced version of reality created by the use of technology to overlay digital information on an image of something being viewed through a device (such as a smartphone camera); also: the technology used to create augmented reality. Merriam Webster Dictionary, retrieved on July 6th 2018 https://www.merriam-webster.com/dictionary/ augmented\%20reality

13. Le Corbusier once described Adolf Loos's Streitschrift Ornament and Crime as a Homeric cleansing. The lecture, first held in the Sophiensaele in Vienna in 1911 castigated the ornament as a "waste of the lifetime of the workers". It can be argued however that this argument has no validity any more as a whole family of computer-controlled machines primarily executes the production of articulation. On the contrary, in the wake of the words full automation it could be argued that the return of ornamentation could provide for jobs, which would otherwise disappear entirely. On a further note, it has to be said that Loos's Argument is entirely engrained in the sentiments of his lifetime, with its respective cultural, economic and political ideas - with no value whatsoever for our contemporary age in which we rather need to investigate changing our relationship to work at large. 